\title{
¿Por qué no soy un terapeuta MINDFULNESS?
}

\author{
$M^{a}$ Luisa Martín Orgilés (1) y Juan Sevillá Gascó (2) \\ (1) Psicóloga. Centro de Terapia de Conducta. Valencia \\ (2) Psicólogo Clínico. Director del Centro de Terapia de Conducta. Valencia
}

\begin{abstract}
resumen/alostract:
En este trabajo se describe con detalle qué es el mindfulness, sus orígenes y evolución, proporcionando una posible explicación de su alta popularidad actual.

Igualmente, se revisa su estatus empírico, llegando a la conclusión de que la investigación no avala su desmesurado uso terapéutico.
\end{abstract}

This article describes in detail what mindfulness is, its origins and development, providing a possible explanation of its high popularity nowadays.

Also, the empirical status of mindfulness is reviewed, concluding that the research does not support its excessive therapeutic use.

\section{nalabras clave/kevwords:}

Mindfulness, Terapia Cognitivo-Conductual, Tercera Generación, Evidencias Empíricas, Análisis Crítico.

Mindfulness, Cognitive-Behavioral Therapy, Third Generation, Empirical Evidence, Critical Analyses.

\section{INTRODUCCIÓN}

Este trabajo tiene diferentes objetivos. El primero de ellos, será describir qué es el mindfulness o consciencia plena, como se tiende a traducir en nuestra lengua. De manera breve, se explicará qué es, su aparición, su ruta histórica hasta llegar al momento presente y algunos de los principales programas de tratamiento que en él se basan.

El segundo objetivo, será revisar la literatura científica, para ver si disponemos de datos que avalen experimentalmente la práctica clínica del mindfulness.

A continuación, el tercer objetivo, más teó- rico, implicará ver la relación que hay entre el mindfulness y la Terapia Cognitivo-Conductual (TCC). Aparentemente es un paradigma nuevo pero, realmente, si desmantelamos la estrategia ¿vamos a encontrar elementos originales?

Finalmente, y como objetivo número cuatro, incluiremos algunas reflexiones sobre el mindfulness $\mathrm{y}$, en general, sobre las terapias de Tercera Generación y algunas recomendaciones para los terapeutas profesionales.

\section{¿QUÉ ES EL MINDFULNESS?}

Hasta hace sólo unas décadas, mindfulness 
era un término desconocido en gran parte de nuestra cultura occidental (Kabat-Zinn, 1982). Pero en los últimos años, los profesionales dedicados al bienestar psicológico estamos asistiendo a un fenómeno creciente: la incorporación de técnicas de meditación oriental al tratamiento de los trastornos emocionales y el consiguiente aumento de publicaciones en la literatura científica que describen dichas intervenciones (Baer, 2003; Germer y otros, 2005; Kabat-Zinn, 2011).

Tal es su grado de expansión, que se ha apuntado como una de las características definitorias de la llamada Tercera Generación de Terapia de Conducta (Hayes, 2004), hablándose incluso de que "podríamos hallarnos ante el umbral de un nuevo modelo de psicoterapia orientado al mindfulness" (Germer y otros, 2005).

Mindfulness nace en la filosofía budista hace 2500 años, para aliviar el sufrimiento humano (Fulton y Siegel, 2005). KabatZinn, pionero y autor de referencia en el trabajo con mindfulness en Occidente, explica que se trata, básicamente, de una forma de prestar atención que consiste en ser consciente de cada instante y lo define como "la consciencia que emerge al prestar atención intencionadamente, en el momento presente y sin juzgar, a cómo se despliega la experiencia momento a momento" (Kabat-Zinn, 2003 , p. 145). Con lo que se espera ser menos reactivo a las experiencias diarias, de forma que el nivel de sufrimiento global se reduzca y aumente la sensación de bienes$\operatorname{tar}$ (Germer y otros, 2005).

En la práctica, se trata de elegir un elemento de la experiencia al que se dirigirá la atención una y otra vez, sirviendo de "ancla" con el momento presente. Este objeto de atención puede ser algo externo (p. e. un sonido), interno (p.e. las sensaciones que produce la respiración), o la experiencia al completo (prestar atención a cualquier fenómeno relevante del momento actual -como la temperatura de la sala, los sonidos, las sensaciones de incomodidad del cuerpo, los pensamientos que van surgiendo...), siendo conscientes de todo lo que sucede, observando nuestros pensamientos, sensaciones y sentimientos, aceptando todo lo que acontezca, sin intentar cambiar nada $\mathrm{y}$, en el momento en que se percibe que la atención se ha desplazado a otro lugar, reconocer dónde se ha ido y devolverla suavemente al objeto de consciencia.

En realidad, esta actividad es algo habitual; en ocasiones, nos sumergimos en una experiencia y la vivimos plenamente (como cuando escuchamos atentamente una melodía o contemplamos una obra de arte), dejándonos llevar, permitiendo que nuestros sentidos se impresionen, siendo conscientes de nuestras reacciones, sin intentar controlar nada. Lo que no es habitual es su continuidad (Germer y otros, 2005). La práctica del mindfulness pretende desarrollar esta capacidad de permanecer en el aquí y ahora.

\section{Principios teóricos.}

El budismo habla de Cuatro Nobles Verdades: 1. La vida está llena de sufrimiento, 2. Éste está causado por el apego a las vivencias agradables y la aversión o evitación de las desagradables, 3. Existe un modo de salir de ese sufrimiento y 4. El camino para ello es seguir el Óctuplo Sendero, que describe 8 comportamientos deseables, entre los que se incluye el mindfulness (Marlatt, 2011).

Kabat-Zinn (1982, 1990) justifica el empleo del mindfulness de la siguiente forma. 
En primer lugar, describe cómo funciona la mente: busca mantener la satisfacción y reducir el malestar. Para lo que constantemente está ideando, planificando, saltando hacia el pasado o hacia el futuro, estresada por cosas que deben hacerse... Es lo que denomina el modo "hacer". Estas reacciones son automáticas, se ponen en marcha casi sin darnos cuenta, especialmente en momentos de crisis, en los que los pensamientos se disparan y buscan rápidamente formas de acabar con ese malestar.

Una de las consecuencias de este funcionamiento de la mente es que, al perderse en el pasado o el futuro, no permanece demasiado tiempo en el presente, por lo que se pierde una gran parte de la riqueza de sus vivencias.

Además, muchas veces no se tiene consciencia de control, al ser testigos simplemente de las reacciones automáticas una vez se han disparado. En este sentido, el mindfulness, o desarrollar el modo "ser", al centrarnos en la experiencia presente podría, por un lado, ayudarnos a vivir de forma más plena nuestras vidas (sin perdernos vivencias por estar "en otro lugar") y, por otro, ser conscientes de cómo se están desencadenando las reacciones automáticas $\mathrm{y}$, desde esa posición de observador privilegiado (al disponer de toda la información del momento presente), tener más libertad para elegir si nos dejamos llevar por ellas o si es más adecuado llevar a cabo una estrategia distinta. En este sentido, diferencia entre "dolor" y "sufrimiento", destacando que ambos estados son naturales (el dolor es ineludible a lo largo de la vida de las personas), pero el sufrimiento es sólo una de las posibles formas de reaccionar ante el dolor, y está condicionado por lo que pensamos acerca de las cosas ("Esto es insoportable”, "No debería pasarme a mí”...).
Esto nos lleva a otro de los puntos fundamentales de la argumentación: "los pensamientos son sólo pensamientos". Nuestra mente está continuamente invadida por un flujo interminable de pensamientos, juicios, puntos de vista ("Soy una mala persona", "Necesito...") resultado de experiencias pasadas que se aplican al presente una y otra vez. Pero en la meditación se tratan todos los pensamientos por igual, con independencia de su contenido, como acontecimientos mentales sin una importancia especial. Por lo tanto, si nuestros pensamientos no tienen por qué ser ciertos, sino que, en muchas ocasiones, son sólo resultado de automatismos, puede ser de gran utilidad aprender una forma de desvincularse de ellos, que permita contemplarlos como un elemento más de lo que experimentamos en un momento determinado (como notar calor, o que la silla en la que estamos sentados sea muy rígida...), pero sin sentirnos en la obligación de seguir sus dictados. De esta forma, además de dejar de sufrir por ellos, se gana objetividad para elegir lo que es más conveniente a la hora de enfrentar una situación en particular. Así, habla de "responder" ante el estrés, más que "reaccionar automáticamente" ante él. Y el camino para ello es a través de la práctica del mindfulness.

Un último aspecto a comentar es el concepto de impermanencia. Un fenómeno que se observa al practicar la consciencia plena es que nada permanece, todo es cambiante (nuestro cuerpo, nuestros pensamientos, sensaciones, emociones, relaciones...), lo que en cierto modo también resulta tranquilizador, dado que una de las ideas que más sufrimiento produce es pensar que estamos en una situación "insoportable" y que ésta "no tendrá fin". Al prestar atención cons- 
ciente, uno se da cuenta de que todo está en continuo movimiento, por lo que ni siquiera en los momentos de mayor desesperación, se está continuamente desesperado.

\section{Evolución del mindfulness en la Terapia Cognitivo-Conductual.}

Aunque nace en el budismo, su práctica es independiente de cualquier sistema de creencias culturales o religiosas (KabatZinn, 1990).

En el campo de la psicoterapia, diversos enfoques teóricos (Terapia de Conducta, Humanista, Sistémica, Psicoanalista...) han utilizado el mindfulness como herramienta subsidiaria a la tecnología existente.

Aquí vamos a repasar los principales hitos en su desarrollo dentro de la Terapia de Conducta.

En 1979 Kabat-Zinn funda la Clínica para la Reducción del Estrés, en el Centro Médico de la Universidad de Massachusetts (hoy conocido como Center for Mindfulness in Medicine, Health Care and Society) y establece el primer programa de entrenamiento de habilidades de consciencia plena para manejar el estrés asociado a enfermedades y trastornos emocionales de naturaleza crónica.

Marlatt, a finales de los 70, empieza a utilizar técnicas de meditación, como una estrategia en la prevención de recaídas en alcoholismo, y en 2005 desarrolla el Programa de Prevención de Recaídas Basado en Mindfulness (Witkiewitz, Marlatt y Walter, 2005).

Hayes, en su Terapia Contextual (1987), reformulada más tarde como Terapia de Aceptación y Compromiso (Hayes, Strosahl y Wilson, 1999) enfatiza la idea de aceptación y utiliza ejercicios de mindfulness para enseñar a las personas a flexibilizar su comportamiento ante las cosas que les hacen sufrir y a perseguir lo que de verdad les importa.

Marsha Linehan (1993a, b), influida por los trabajos anteriores, tomó prestadas algunas de las ideas de la filosofía Zen para desarrollar su Terapia Dialéctica Conductual, originalmente concebida para el tratamiento del Trastorno Límite de Personalidad. Otorga un papel relevante a las habilidades de consciencia plena.

Wells y Matthews (1994, 1996), en su Terapia Metacognitiva, han sugerido el uso de estrategias de mindfulness para que los individuos puedan llevar a cabo un distanciamiento metacognitivo de los pensamientos: aumentando el control sobre la atención para desconectarse de la rumiación y liberando así los recursos atencionales que se necesitan para cambiar las creencias desadaptativas.

Segal, Williams y Teasdale elaboran la $T e$ rapia Cognitiva Basada en la Consciencia Plena (2002), que representa el primer programa desde la Terapia Cognitivo-Conductual para la prevención de recaídas en depresión.

Y, más recientemente, se están planteando numerosos proyectos como la Terapia Cognitivo-Conductual Basada en Mindfulness para Trastornos de Adicción en comorbilidad con Trastornos del Estado de Ánimo (Hoppes, 2006), la Práctica de Alimentación Consciente Basada en Mindfulness (MB-EAT) para trastornos de alimentación (Kristeller, Baer y Quiliam-Wolever, 2006), la Terapia Conductual Basada en la Aceptación para el Trastorno de Ansiedad Generalizada (Roemer y Orsillo, 2007), la Terapia de Grupo Basada en la Aceptación 
y el Mindfulness (MAGT) para Trastornos de Ansiedad Social (Kocovski, Fleming y Rector, 2009)... por citar algunos.

Hasta el momento se ha empleado para tratar todo tipo de problemas de ansiedad, insomnio, depresión, trastorno bipolar, problemas sexuales, de pareja, alimentación, adicciones, fatiga crónica, psicosis, personalidad...

En función del protagonismo que se otorga al entrenamiento de habilidades específicas de mindfulness se ha diferenciado entre las Intervenciones Basadas en el Entrenamiento en Mindfulness -como la Reducción del Estrés Basado en la Consciencia Plena- y las Intervenciones que Incorporan el Entrenamiento en Mindfulness -como la Terapia Dialéctica Conductual o la Terapia de Aceptación y Compromiso (Baer, 2003).

\section{Aplicaciones clínicas.}

A continuación, vamos a describir algunos de los principales programas, citados arriba.

\section{Reducción del Estrés Basada en la Cons- ciencia Plena - REBCP (Kabat-Zinn, 1982):}

Todas las estrategias que se enseñan en este programa cultivan mindfulness de forma específica (Germer y otros, 2005). Se realizan ejercicios de: exploración corporal, consistente en un repaso mental del cuerpo para aumentar la consciencia en cada zona; meditación basada en la respiración, en el cuerpo, en la consciencia y en los sonidos, empleando estiramientos y la marcha consciente; y yoga, para mejorar la flexibilidad y realizar un completo trabajo respiratorio mediante posturas concretas. Todo esto se combina con diálogo, psicoeducación sobre el estrés, lectura de poesía, historias... y tareas para casa de 45 minutos diarios 6 veces por semana. El programa se desarrolla en 8 sesiones de 2 horas y media, una por semana, en grupos de hasta 30 personas; se suele añadir una sesión de día completo tras la sesión número 6. La finalidad es entrenar una actitud mental de observación, de apertura a la incomodidad y acabar con los hábitos mentales que, en un intento precipitado por hacer desaparecer el malestar, aumentan el estrés e impiden un manejo adecuado de la situación. En las 3 últimas décadas más de 18.000 personas han seguido el programa en todo el mundo y ha sido objeto de numerosos estudios para poner a prueba su eficacia.

Terapia Cognitiva Basada en la Consciencia Plena - TCBCP (Segal, Williams y Teasdale, 2002):

Segal, Williams y Teasdale, mientras estudiaban el proceso de recaída en clientes que habían superado una depresión, vieron en el mindfulness una herramienta que podría ayudar a estas personas a desvincularse de las reacciones mentales que se ponen en marcha de manera automática cuando el estado de ánimo empieza a bajar de forma sutil y que pueden desencadenar un nuevo episodio depresivo. Así, desarrollando habilidades de consciencia plena se podría: detectar de forma temprana cuándo su estado de ánimo empieza a cambiar, observar sus pensamientos negativos como eventos mentales y no como reflejos fieles de la realidad, centrándose en la experiencia presente, ocupar el espacio destinado a la rumiación -impidiendo así que ésta se instaure-, tolerar el malestar sin la necesidad de reaccionar impulsivamente a él y desde esa postura de distanciamiento, poder elegir la mejor acción para abordar la situación en la que se encuentran. Se toma del 
programa de Kabat-Zinn el formato grupal (aunque con un máximo de 12 participantes), la duración de las sesiones, los ejercicios de meditación, tener consciencia y registrar acontecimientos agradables y desagradables, lectura de poemas que apoyan los contenidos comentados en las sesiones, videos del programa REBCP, lecturas, las tareas para casa... Y de la Terapia Cognitiva se toma la psicoeducación sobre depresión, el Cuestionario de Pensamientos Automáticos de Hollon y Kendall (1980), establecer las relaciones ente actividades agradables y estado de ánimo, programar actividades agradables, de satisfacción y dominio, la explicación del $\mathrm{ABC}$, el cuestionamiento de los pensamientos, la identificación de las señales de recaída de depresión y cómo actuar ante ellas... Hoy en día, en Reino Unido este programa es recomendado por la NICE (Nacional Institute for Clinical Excellence) para prevenir la depresión en aquellas personas que han tenido 3 o más episodios previamente.

\section{Terapia Dialéctica Conductual - TDC (Linehan, 1993):}

Marsha Linehan desarrolló una Terapia Cognitivo-Conductual para tratar a clientes con Trastorno Límite de la Personalidad (TLP), especialmente difíciles, que no mejoraban con las técnicas tradicionales. Esta terapia se desarrolla en cuatro fases. En la primera, se aborda el descontrol conductual grave durante un año; las metas son reducir las conductas suicidas y parasuicidas, los comportamientos que interfieren tanto en la terapia como en la calidad de vida en general y aumentar las habilidades psicosociales (de consciencia, regulación emocional, tolerancia al malestar e interpersonales); esto se hace en sesiones semanales, tanto individuales (de una hora), como en grupo (2 horas y media) y a través de contactos telefónicos. En las siguientes fases se trabaja el estrés postraumático, el respeto por sí mismo y el planteamiento de metas vitales, y finalmente, se acomete el desarrollo de un sentimiento de satisfacción sostenido. Linehan redefinió el TLP como un patrón de inestabilidad y desregulación emocional, interpersonal, conductual, cognitivo y del sentido del yo, y desarrolló la Teoría Biosocial para explicar el origen de estos trastornos, que serían el resultado de la interacción entre una determinada predisposición biológica y un ambiente invalidante. Adopta una visión dialéctica del mundo, según la cual la realidad es un conjunto dinámico de fuerzas opuestas y las respuestas extremas, características del TLP, serían fracasos a la hora de conseguir una síntesis entre ambas polaridades. La idea de incorporar habilidades de mindfulness surge del intento de resolver una de las principales dialécticas en las que se ven inmersas estas personas: la resistencia a cambiar y a seguir como están. La síntesis de estos 2 opuestos se logra al integrar estrategias de cambio (TCC) con estrategias de aceptación (mindfulness). De las habilidades que se enseñan en la primera fase de la terapia, las de consciencia plena son las más importantes; a través de ellas, se persigue la regulación del yo, que la persona sea capaz de experimentarse a sí misma. Define el mindfulness en términos de Habilidades Qué (Describir, Observar, Participar) y Habilidades Cómo (No emitir juicios, Unicidad mental, Efectividad) para su comprensión y entrenamiento. También utiliza el mindfulness, especialmente, en las habilidades de tolerancia a las crisis, como estrategia de aceptación.

Terapia de Aceptación y Compromiso - 


\section{ACT(Hayes, Strosahl y Wilson, 1999):}

Se basa en la Teoría del Marco Relacional (Hayes, Barnes-Holmes y Roche, 2001), que da cuenta de los mecanismos que rigen el lenguaje y la cognición, para desarrollar técnicas que puedan manipular el comportamiento humano. Entiende que la sociedad transmite la idea de una normalidad destructiva, al proclamar que la felicidad es la ausencia de malestar. Esto es imposible dado que somos biológicos y verbales (continuamente evaluamos, comparamos, categorizamos, añadimos contenidos $\mathrm{y}$ emociones, revivimos el pasado, fabricamos futuros...). Pero cuando las personas, influidas por esas ideas, se empeñan en no sufrir se puede desarrollar un patrón de funcionamiento general basado en intentar librarse de la incomodidad, el Trastorno de Evitación Experiencial (TEE), y que es propuesto como un sistema alternativo de clasificación de los trastornos emocionales. La meta fundamental de esta terapia será generar flexibilidad psicológica para alterar esa Evitación Experiencial Destructi$v a$, que aleja a las personas de la vida que realmente querrían vivir si no estuvieran ocupadas en intentar no sufrir. Para lograrlo, desde el principio se esclarecen cuáles son sus valores, ya que ellos serán la razón por la cual serán capaces de quedarse en contacto con la aversión. A lo largo de la terapia se pretende reducir la influencia de las ideas que la cultura ha expandido acerca de la naturaleza de la felicidad; se hace ver al cliente cómo sus intentos de eliminar el sufrimiento han sido inútiles, que estos han sido, precisamente, el problema (el intento de control); y como alternativa, se presenta la aceptación, se anima a adoptar una actitud de contemplación ante las reacciones emocionales y los pensamientos, minimizando su importancia. Con todo esto no se pretende que el malestar desaparezca, sino que al cliente le vaya mejor en la vida. Como métodos utiliza metáforas, paradojas y ejercicios experienciales. El papel del mindfulness queda reflejado en ejercicios de contemplar sin reaccionar, en el entrenamiento para renunciar al control.

\section{¿Qué beneficios aporta el entrenamiento de la consciencia plena?}

Muchas son las bondades que se han atribuido al desarrollo de este tipo de atención (Reiman, 1985; Kabat-Zinn, 1990; Valentine \& Sweet, 1999; Segal, Williams y Teasdale, 2002; Davidson et al, 2003; Germer, Siegel y Fulton, 2005; Morgan y Morgan, 2005; Grossman, Niemann, Schmidt y Walach, 2004; Cebolla y Miró, 2008).

Entre los beneficios clínicos para los clientes, se ha dicho que el entrenamiento de la atención plena: disminuye el estrés, produce relajación, permite disfrutar más de las cosas, aumenta la tolerancia a la aversión, rebaja el dolor físico e incrementa la capacidad para manejarlo, reduce el nivel global de sufrimiento y la sensación de bienestar aumenta, ayuda a salir del proceso de rumiación y ver las cosas tal como son, disminuye la frecuencia de la rumiación cognitiva, favorece el percatarse de los cambios emocionales, suaviza las interpretaciones, cambia la relación con la ansiedad desde la evitación temerosa a la tolerancia (se aprende la no-evitación hasta que el miedo disminuye), produce ecuanimidad, lleva a una mayor libertad psicológica y emocional, mejora la autoestima, aumenta la capacidad de percibir las señales emocionales no verbales de los otros, así como la de "sentir sus mundos interiores"... 
También se han destacado efectos positivos para los terapeutas que la practican: se sienten más "presentes", relajados y receptivos con sus clientes (Germer y otros, 2005), puede aumentar la atención indirecta "vía insights intuitivos", fomenta un estado de reflexión y disponibilidad emocional que se dice es muy útil para la labor clínica, cultiva numerosas cualidades beneficiosas para establecer una fuerte relación terapéutica: atención, compasión, empatía, aceptación, una perspectiva más amplia del sufrimiento...

Y otras ventajas no clínicas, como: se ralentiza la percepción del tiempo, genera energía y entusiasmo por la vida, clarividencia y alegría, refuerza la capacidad de la mente de permanecer en cualquier objeto mental de una forma concentrada y sostenida, mejora los procesos de curación y la respuesta inmunitaria...

Se han dicho muchas cosas, pero ¿qué revelan realmente los datos de que disponemos? ¿Es realmente cierto todo esto? ¿Está plenamente justificado el uso masivo del entrenamiento de esta forma de relacionarse con la experiencia?

\section{EVIDENCIAS EMPÍRICAS SOBRE LA EFICACIA DEL MINDFULNESS}

Este apartado no es una revisión sistemática ni tampoco un metaanálisis formal sobre la eficacia del mindfulness, sino una evaluación crítica de los distintos metaanálisis publicados.

Desde principios del año 2000 hasta la actualidad se han publicado unos cuantos metaanálisis, entre los que destacan: Bishop (2002), Baer (2003), Grossman, Niemann, Schmidt y Walach (2004), Torneatto y Nguyen (2007), Chiesa y Serreti (2009),
Zgierska, Rabago, Chawla, Kushner, Kohler y Marlatt (2009), Hoffman, Sawyer, Witt y Oh. (2010) y Miró, Perestelo-Pérez, Rivero, González, De la Fuente y Serrano (2011).

Excepto el trabajo de Bishop (2002) y Torneatto y Nguyen (2007) cuyas conclusiones son que no hay soporte empírico para la eficacia del mindfulness (y que han sido recientemente criticados por sus singularidades), el resto de los metaanálisis concluyen que "el mindfulness es una aportación prometedora".

Se usan expresiones como: "moderada evidencia", "puede ser de ayuda", o usando terminología del Task Force del APA (1995), el mindfulness estaría en la categoría de técnica "probablemente eficaz".

Todos estos trabajos (también) ponen de manifiesto dos hechos. El primero es que en las búsquedas sistemáticas aparecen cientos de referencias sobre el mindfulness. Lo que es una clara evidencia de cuán de moda está. Todo el mundo publica sobre el tema. Sin embargo, la mayoría de las publicaciones no tienen ningún interés científico por su carencia de rigor. Segundo, de los estudios aceptados, con criterios, en algunos casos, poco exigentes, la mayoría de los trabajos tienen una importante debilidad metodológica: muestras demasiado pequeñas, ausencia de grupo control o lista de espera, poblaciones muy dispares, instrumentos de evaluación débiles, falta de control de otras variables, seguimientos cortos, efectos del tamaño bajos... etc. En todas las revisiones citadas se insiste en la falta de rigor de los estudios disponibles y se hace un llamamiento a la necesidad de futuras investigaciones metodológicamente sólidas; muchas de ellas también señalan la falta de estudios comparativos con las técnicas ya demostradas eficaces. 
Además hay otro tipo de problema, respecto a los protocolos aplicados. Los estudios evaluados incluyen, al menos: REBCP, TCBCP y TDC. Estos enfoques terapéuticos, además de las estrategias mindfulness, incluyen otras técnicas puramente cognitivo-conductuales, desde la Exposición, el Entrenamiento de Habilidades Sociales, la Relajación y la Terapia Cognitiva. ¿Cómo establecer la causalidad de los resultados obtenidos? ¿Qué ingredientes son activos?

A modo de conclusión, se puede afirmar que estamos aún en un período precientífico en términos de la eficacia del mindfulness. Sería necesario, por lo tanto, una mayor cantidad de estudios con una metodología rigurosa. Afinar y definir qué es el mindfulness, para sacar conclusiones sólidas acerca de su utilidad y eficacia. Y contrastarlo con las tecnologías ya validadas.

\section{MINDFULNESSY TERAPIA COGNITIVO-CONDCUTUAL}

Aparentemente el mindfulness es una estrategia nueva, o mejor dicho, es una estrategia con una historia larga, pero que en la actualidad está de moda. Si la comparamos con el repertorio de técnicas cognitivo-conductuales, parece original, diferente, nueva ¿pero es así? Siguiendo el excelente trabajo de Baer (2003) y otros parecidos, y nuestras propias reflexiones teóricas, a continuación vamos a analizar lo que llamamos mindfulness, desmantelándolo, seccionándolo en procesos psicológicos mucho más claros conceptualmente y con los que todos los terapeutas cognitivo-conductuales estamos familiarizados.

\section{Exposición.}

La práctica del mindfulness implica focalizar y mantener la atención en un determina- do estímulo con una actitud de aceptación y no evaluación ante cualquier fenómeno psicológico que pueda aparecer: pensamientos, emociones o sensaciones fisiológicas. Además, mantener estos comportamientos durante períodos prolongados de tiempo.

Si se mantiene la atención sobre un pensamiento asociado a la ansiedad, o a una sensación fisiológica también conectada a la ansiedad, sin permitir la ejecución de conductas de escape, inevitablemente se producirá la habituación al pensamiento/ sensación y la consiguiente extinción de la ansiedad.

Y como el lector sabe, estas estrategias de Exposición a los pensamientos o Exposición a las sensaciones fisiológicas son el centro del enfoque terapéutico para el Trastorno Obsesivo Compulsivo, el Trastorno de Ansiedad Generalizada, el Trastorno de Pánico, el Trastorno de Estrés Postraumático y la Hipocondría (Abramowitz, Deacon, y Whiteside, 2011).

Desde este punto de vista, el mindfulness sería una forma diferente, aunque quizás no tanto, de enseñar y practicar Exposición a los pensamientos y/o Exposición Interoceptiva.

\section{Terapia Cognitiva.}

Sí, el mindfulness implica modificaciones cognitivas, cambio cognitivo. Terapia Cognitiva.

En nuestra opinión, y como apuntan Segal et al (2002), la habilidad más potente que se puede aprender en el mindfulness es la habilidad metacognitiva. Es decir, ser capaz de diferenciar los pensamientos del propio yo.

Aunque la Terapia Cognitiva más estándar implica analizar el contenido de los pensa- 
mientos, también es una práctica frecuente, en momentos avanzados de la terapia, etiquetar los pensamientos perturbadores como lo que son, sólo pensamientos. Esta habilidad no sólo se usa en trastornos de Ansiedad (Clark y Beck, 2010) o Depresión (Wells, 2009) sino en problemas de otro calibre como la Esquizofrenia (Beck, Rector, Stolar y Grant, 2009) o el Trastorno Bipolar (Lam, Jones, Hayward y Bright., 2012).

Todavía más, la propia Terapia Cognitiva, se basa en el desarrollo de la metacognición. En esa línea y citando propuestas más recientes, destacaríamos la Terapia Metacognitiva del profesor Adrian Wells (2009) como un paradigma refrescante y prometedor, aunque aún no validado.

\section{Autocontrol.}

La práctica constante del mindfulness a través de la meditación, probablemente, aumenta la habilidad de autoobservación. Y como el lector sabe, la autoobservación es el primer paso para el autocontrol o el autogobierno.

Esta habilidad de autorrastreo podría utilizarse para notar los primeros pasos de una recaída en un trastorno adictivo (Marlatt, 1994) o ante un episodio depresivo (Segal y otros, 2002) y actuar en consecuencia, usando habilidades de control previamente adquiridas, para alterar el curso de los acontecimientos.

O, como apunta Linehan (1993a, 1993b), esta clara percepción de las emociones o pensamientos propios podría ser el primer peldaño para ejercer control sobre el exceso conductual característico del Trastorno Límite de la Personalidad.

\section{Relajación.}

Aunque de una forma directa y como objetivo, el mindfulness no intenta conseguir un estado de relajación, no hay duda de que, indirectamente al menos, se consigue. Basta con revisar algunas de las prácticas (Kabat-Zinn, 1990; Segal y otros, 2002)

La conclusión indiscutible de este análisis del concepto mindfulness es que su ejecución, de forma indirecta, incluye la aplicación de toda una serie de estrategias, sobradamente validadas, dentro de la TCC. ¿Significa esto que el mindfulness es TCC?

Probablemente no, más bien que tiene una serie de propuestas en común, pero también diferencias.

Las principales diferencias tienen que ver con el objetivo. En el mindfulness el cambio que se persigue es indirecto y general. Se trata de desarrollar una actitud distinta ante la dificultad (especialmente) de no reactividad, de aceptación, de espectador sobre las propias emociones, pensamientos o sensaciones, sin evaluar o juzgar, de manera amable -como se suele decir en el argot-, que rompa las reacciones automáticas, precipitadas y, en muchas ocasiones, contraproducentes de intentar desembarazarse cuanto antes de la incomodidad. Para ello, se entrena al individuo en meditar $\mathrm{y}$, probablemente, esto produce o tiene importantes elementos de Exposición, TC, Automanejo y Relajación, pero estos no son los objetivos explícitos.

En TCC también se busca cambiar esas respuestas inadecuadas del cliente, que son responsables del mantenimiento de los problemas, pero de forma más directa, estableciendo objetivos de trabajo claros y a través del entrenamiento específico en técnicas validadas, para ganar autocontrol. 


\section{REFLEXIONES, RECOMENDACIONES $Y$ CONCLUSIONES}

El mindfulness está de moda. Basta con ver el número de publicaciones, cursos, simposiums que sobre el mismo se ofertan (por citar un ejemplo reciente, en el pasado 41 Congreso Anual organizado en Reykjavik por la European Association for Behavioural and Cognitive Therapies, la presencia de comunicaciones relacionadas con el mindfulness en temas como depresión, trastorno bipolar, manejo del dolor, adicciones, TPL... fue más que notable (por encima del 25\%). Desde hace unos pocos años, el interés de los terapeutas cognitivoconductuales y de otras orientaciones por el mindfulness ha aumentado exponencialmente. Pero, ¿está realmente justificado? Pensamos que no.

¿Por qué todo el mundo se siente fascinado por el mindfulness? Creemos que se debe a una combinación de variables. Primero, por el efecto de atracción que genera la cultura oriental. En occidente tendemos a sentirnos atraídos por las filosofías y culturas orientales. Pensar en oriente está asociado a armonía, sabiduría, control, saber vivir. Inmediatamente nos imaginamos a un lama tibetano, en postura del loto, meditando al amanecer con el magnífico Himalaya a sus espaldas. Este apetito por lo oriental se mantiene constante y aumenta cíclicamente nutriendo al arte, moda, gastronomía, decoración y, cómo no, al autocontrol. En esta línea, algunos autores señalan que quizá muchos de los jóvenes que en los 60 y 70 participaron del movimiento de "contracultura" en el que la meditación estaba a la orden del día (especialmente en la sociedad norteamericana) hoy son investigadores y clínicos que mantienen ese foco de interés y lo plasman en sus trabajos (Siegel, Germer y Olendzki, 2010).

Una segunda variable sería una especie de postura pendular o de rebote ante el cognitivismo que lleva reinando desde hace tres décadas. Este fenómeno explicaría, además o especialmente, la aparición de muchas de las terapias de Tercera Generación, sobre todo, la Terapia de Aceptación y Compromiso, construida a partir del Conductismo radical. Y es comprensible. Para muchos académicos y practicantes, el Cognitivismo se ha convertido en una escuela teórica diferente al Conductismo. Se ha perdido el énfasis en el análisis funcional a favor del uso de clasificaciones psiquiátricas estandarizadas, se han medicalizado los trastornos psicológicos convirtiéndolos en enfermedades y se han dicho afirmaciones tan atrevidas, mantra habitual del Cognitivismo, como que los problemas emocionales se mantienen por los Pensamientos Negativos Automáticos.

Sin embargo, en nuestra opinión, la solución no es volver a los años 70 en cuanto a desarrollo de la ciencia y negar el papel funcional de los pensamientos. Por supuesto que los pensamientos no mantienen los trastornos psicológicos, pero sí forman parte de una cadena de acontecimientos y eventos psicológicos, con relación de causa y efecto, que desembocan en la conducta operante que provoca las consecuencias, que sí mantienen el problema (Sevillá y Pastor, 1997).

Algunos creemos que la TCC es la evolución científica del Conductismo original y de la Terapia de Conducta. No creemos en dos modelos diferentes, hablamos de la misma ciencia madre. Una ciencia que tiene un modelo teórico robusto y una metodología de trabajo sólida. 
Por otra parte, tampoco se puede borrar de un plumazo la eficacia de la TCC. Tenemos cientos de estudios en todos los campos, incluido el de las psicosis, de que estos tratamientos funcionan. En nuestra opinión, más por los elementos conductuales que incluyen, que por los puramente cognitivos. Lo que no significa que el método sea inadecuado. Muchas veces, la mejor manera de conseguir cambiar las operantes es modificando previamente las respondentes cognitivas que la anteceden. Y para esto está la investigación, para decirnos lo que es más eficaz en cada caso. Por ejemplo, en el campo de los trastornos de Ansiedad, hay problemas en que añadir Terapia Cognitiva aumenta la eficacia de la Exposición, y en otros, sin embargo, no.

Nos llama la atención algunas aseveraciones que se han publicado en el marco de las terapias de Tercera Generación, especialmente desde la Terapia de Aceptación y Compromiso (Hayes, Strosahl y Wilson, 1999) y la Psicoterapia Analítico Funcional (Kohlenberg y Tsai, 1991). Nos referimos a las críticas sobre la Terapia Cognitivo-Conductual que se han hecho desde estos paradigmas. Creemos que son críticas poco fundamentadas $y$ que demuestran un conocimiento superficial del tema. Por ejemplo, que el objetivo de la Terapia Cognitivo-Conductual es sustituir pensamientos "negativos" por "positivos", enseñar a la persona a pensar de otro modo, a suprimir el contenido desagradable de algunos pensamientos o a eliminar recuerdos desagradables (Wilson y Luciano, 2002).

Nada de esto pensamos que sea cierto. La Terapia Cognitivo-Conductual tiene un repertorio de técnicas amplio. Algunas, efectivamente, se centran en modificar directamente ciertos pensamientos para, a través de esta modificación, cambiar la conducta operante, y otras, cuyo objetivo es cambiar directamente la conducta operante. El ejemplo más habitual del primer grupo son las técnicas verbales que, a menudo, se conocen como Reestructuración Cognitiva. Del segundo grupo, la técnica más genuina es la Exposición, que es la base del tratamiento Cognitivo-Conductual de todos los trastornos de Ansiedad.

Las técnicas verbales se centran en analizar la veracidad o no de una determinada interpretación ("Soy un inútil”) y si el resultado de ese examen demuestra que es un pensamiento no realista, entonces se busca una alternativa sustentada por los datos, realista ("He cometido un error"), no positiva ("No pasa nada, soy muy listo"), y esa nueva forma de pensar, se usa para cambiar la operante motora.

No se trata de enseñar a pensar de "otro modo" en general, sino de crear el hábito de analizar aquellos pensamientos que son parte del dolor psicopatológico, contrastar su inadecuación e intentar ser más realista $y$, de esta forma, facilitar que la persona actúe de la manera más conveniente ante las situaciones difíciles de su vida (como cuando en fobia social enseñamos a desdramatizar los pensamientos acerca de una ejecución catastrofista, para que la persona se atreva a levantar la mano y preguntar en público o mantenga una conversación, para que pueda comprobar que sus temores no estaban justificados y esté en contacto con las contingencias naturales derivadas de la actuación acorde con la situación).

No se huye de los pensamientos de contenido desagradable o los recuerdos traumáticos. Todo lo contrario. En trastornos como el Estrés Postraumático, algunos tipos de Trastorno Obsesivo-Compulsivo o en el 
Trastorno de Ansiedad Generalizada, el tratamiento de elección es la Exposición a los pensamientos, bien en formato de imagen o de contenido verbal. La manera de dejar de sufrir por un elemento cognitivo doloroso es exponerse a él, habituarse, y que la emoción asociada, habitualmente ansiedad, se extinga.

El lector interesado en profundizar a las réplicas ante las críticas de líderes de terapias de Tercera Generación sobre la Terapia Cognitivo-Conductual encontrará muy educativo el magnífico trabajo de Hofman y Asmundson (2008).

Nosotros también tenemos dudas sobre la Terapia de Aceptación y Compromiso y las vamos a formular planteando interrogantes.

Por ejemplo, la Teoría del Marco Relacional (Hayes, Barnes-Holmes y Roche, 2001), que nos parece un modelo brillante y con el que, esencialmente estamos de acuerdo, ¿aporta mucho al trabajo de Skinner (1957) sobre la adquisición del lenguaje?

¿Puede considerarse predictiva una (mera) propuesta de clasificación de los Trastornos Emocionales aquélla que habla tan sólo de una categoría, el Trastorno de Evitación Experiencial (Hayes, Wilson, Gifford, Follete y Strosahl, 1996; Luciano y Hayes, 2001)?

El concepto de Trastorno de Evitación Experiencial ¿aporta mucho más a conceptos como: baja tolerancia a la frustración, dificultad para retardar el refuerzo o falta de autocontrol o, simplemente, conductas mantenidas por refuerzo negativo?

¿No es parte esencial de la naturaleza humana buscar el placer y huir del dolor? Quizás el problema no sea éste, sino qué placer se busca, qué dolor se evita y cuándo y cómo se hace esto.

¿No resulta curioso y contradictorio que la Terapia de Aceptación y Compromiso critique el cambio cognitivo y se centre en, precisamente, cambiar los valores que, obviamente, son pensamientos y creencias?

En otro orden de cosas, ¿a alguno de los autores de referencia del mindfulness se le ha ocurrido pensar que, si la mente humana tiene una extraordinaria capacidad para desfocalizar del presente y retroceder al pasado, o proyectarse hacia el futuro, será por algo? ¿No será un mecanismo adaptativo encaminado a sobrevivir aumentando las probabilidades de encontrar soluciones eficaces a los problemas que nos toca vivir?

Y en esta línea, ¿sería bueno desconectar las emociones de los pensamientos? ¿No nos dejaría este proceso en una postura de vulnerabilidad ante los peligros reales de la vida? ¿Cómo sabríamos de qué pensamientos fiarnos y de cuáles no?

\section{Concluyendo.}

Nos resulta sorprendente comprobar cómo, en estos años, pocas voces se hayan alzado con una visión crítica hacia el mindfulness y hacia algunos aspectos de las nuevas psicoterapias.

La realidad es que, desde un punto de vista experimental, estamos aún en una etapa muy básica. Si como terapeutas cognitivoconductuales uno de nuestros objetivos prioritarios es ofrecer a nuestros clientes tratamientos que "sabemos" que funcionan (empíricamente validados) más que tratamientos que "creemos" que funcionan, entonces no podemos ofrecer el mindfulness como una opción. Al menos todavía no. Es muy probable que esto ocurra en un futu- 
ro relativamente próximo, pero aún no. $\mathrm{O}$ quizás ocurra como lo que pasó con las Terapias Constructivistas en los años 90, de las que ahora parece que nadie habla. El tiempo lo dirá.

En términos más conceptuales o teóricos, si se prefiere la expresión, si el mindfulness parece que provoca el uso de procedimientos bien establecidos como la Exposición, la Terapia Cognitiva, el Autocontrol o la Relajación, y en el caso de funcionar, lo hiciera a través de estas estrategias, nuestra opinión es que las usemos directamente. Primero porque sabemos cómo usarlas de la manera más efectiva $\mathrm{y}$, segundo, porque tenemos una acumulación enorme de evidencias sobre su eficacia.

Por otro lado, no podemos obviar las diferencias culturales: pedir a la gente que medite 45 minutos diarios tiene sentido en Kyoto pero menos en Teruel. Siempre habrá gente que sintonice con estas ideas, pero no nos engañemos, a muchos les resultará ajeno. Así como parte del léxico empleado: paz interior, armonía, sanación, clarividencia, inmovilismo, espiritualidad, sabiduría inherente, dharma, impermanencia... Términos que nos transportan a otras esferas que suenan de manera muy distinta a como lo hacen las Ciencias de la Salud.

Por todo esto, apostamos por una óptica algo más crítica frente a un fenómeno que se ha extendido de manera poderosa y, en nuestra opinión, sin una base sólida hasta el momento que lo avale. Si bien es cierto que hay muchos estudios que arrojan datos prometedores y experiencias alentadoras, no podemos dejar de reclamar cautela, rigor $y$, como clínicos, no precipitarnos a emplear un método que puede resultar atractivo sin plantearnos realmente qué aporta a lo que ya estamos utilizando y si su uso está justificado.

\section{Referencias}

Abramowitz, J. S., Deacon, B. J. y Whiteside, S. P. (2011) Exposure therapy for Anxiety. Principles and Practice. New York: The Guilford Press.

Baer, R. A. (2003) Mindfulness training as a clinical intervention: a conceptual and empirical review. Clinical Psychology: Science and Practice, 10, 125-143.

Beck, A. T., Rector, N. A., Stolar, N. y Grant, P. (2009) Schizophrenia. Cognitive Theory, Research and Therapy. New York: The Guilford Press.

Bishop, S. R. (2002) What do we really know about Mindfulness-Based Stress Reduction? Psychosomatic Medicine, 64, 71-84.

Bowen, S., Chawla, N. y Marlatt, G. A. (2011) Mindfulness-Based Relapse Prevention for addictive behaviors. A clinician's guide. New York: The Guilford Press.

Cebolla, A. y Miró, M. T. (2008) Efectos de la Terapia Cognitiva basada en la Atención Plena: una aproximación cualitativa. Apuntes de Psicología, 26 (2), 257-268.

Chiesa, A. y Serretti, A. (2009) Mindfulness-Based Stress Reduction for Stress Management in healthy people: a review and meta-analysis. The Journal of Alternative and Complementary Medicine, 15 (5), 593-600.

Clark, D. H. y Beck, A. T. (2010) Cognitive Therapy of Anxiety Disorders. Science and Practice. New York: The Guilford Press.

Davidson, R., Kabat-Zinn, J., Schumaher, J., Rosenkranz, M., Muller, D., Santorelli, S., Urbanowski, F., Harrington, A., Bonus, K. y Sheridan, J. F. (2003) Alterations in brain and immune function produced by mindfulness meditation. Psychosomatic Medicine, 66, 149-152.

Fulton, P. R. y Siegel, R. D. (2005) Buddhist and westwern psychology. Seeking common ground. En Germer, C. K., Siegel, R. D. y Fulton, P. R. (Eds) Mindfulness and psychotherapy. New York: The Guilford Press.

Germer, C. K., Siegel, R. D. y Fulton, P. R. (Eds) (2005) Mindfulness and psychotherapy. New York: The Guilford Press.

Grossman, P., Niemann, L. Schmidt, S. y Walach, H. (2004) Mindfulness-Based Stress Reduction and health benefits. A meta-analysis. Journal of Psychosomatic Research, 57, 35-43.

Hayes, S. C. (1987) A contextual approach to therapeutic change. En Jacobson, N. S. (Ed.), Psychotherapists in clinical practice: cognitive and behavioural perspectives. New York: The Guilford Press.

Hayes, S. C. (2004) Acceptance and commitment therapy, 
relational frame theory, and the third wave of behavioural and cognitive therapies. Behavior Therapy, 35, 639-665.

Hayes, S. C., Barnes-Holmes, D. y Roche, B. (Eds) (2001) Relational Frame Theory A post-skinnerian account of human language and cognition. New York: Kluwer Academic.

Hayes, S. C., Strosahl, K. D. y Wilson, K. G. (1999) Acceptance and commitment therapy: an experiential approach to behaviour change. New York: The Guilford Press.

Hayes, S. C., Wilson, K. G., Gifford, E. V., Follete, V. M. y Strosahl, K. (1996) Experiential Avoidance and Behavior Disorder: A functional dimensional approach to diagnosis and treatment. Journal of Consulting and Clinical Psychology, 64, 1152-1168.

Hofman S. G. y Asmundson, G. (2008) Acceptance and mindfulness-based therapy: New wave or old hat? Clinical Psychology Review, 28 (1), 1-16.

Hofman, S. G., Sawyer, A. T., Witt, A. A. y Oh, D. (2010) The effect of mindfulness-based therapy on anxiety and depression: a meta-analytic review. Journal of Consulting and Clinical Psychology, 78 (2), 169-183.

Hoppes, K. (2006) The application of mindfulness-based cognitive interventions in the treatment of co-occurring addictive and mood disorders. CNS Spectrum, 11, 829851.

Kabat-Zinn, J. (1982) An outpatient program in behavioral medicine for chronic pain patients on the practice of mindfulness meditation: Theoretical considerations and preliminary results. General Hospital Psychiatry, 4, 33-47.

Kabat-Zinn, J. (1990) Full catastrophe living. New York: Delacorte.

Kabat- Zinn, J. (2003) Mindfulness-based interventions in context: past, present, and future. Clinical Psychology: Science and Practice, 10, 144-156.

Kabat- Zinn, J. (2011) Prefacio. En Didonna, F. (Ed), Manual clínico de mindfulness. Bilbao: Desclée de Brouwer.

Kristeller, J. L., Baer, R. A. y Quillian-wolever, R. (2006) Mindfulness based-approaches to eating disorders. En Baer, R. (Ed) Mindfulness and acceptance-based interventions: Conceptualization, application, and empirical support. San Diego: Elsevier.

Kocovski, N. L., Fleming, J. y Rector, N. A. (2007) Mindfulness and acceptance-based group therapy for social anxiety disorder: An open trial. Cognitive and Behavioral Practice, 16 (3), 276-289.

Kohlenberg, R. J. y Tsai, M. (2007) FAP. Psicoterapia Analítica Funcional. Creación de relaciones intensas y curativas. Málaga: Servicio de Publicaciones de la Universidad de Málaga.

Lam, D. H., Jones, S. H., Hayward, P. y Bright, J. A. (2012)
Cognitive Therapy for Bipolar Disorders. Londres: WilleyBlackwell.

Linehan, M. M. (1993a) Cognitive-Behavioral Treatment of Borderline Personality Disorder. New York: The Guilford Press.

Linehan, M. M. (1993b) Skills Trainig Manual for Treating Borderline Personality Disorder. New York: The Guilford Press.

Luciano, M. C. y Hayes, S. C. (2001) Trastorno de Evitación Experiencial. International Journal of Clinical and Health Psychology, 1, 109-157.

Marlatt, G. A. (1994) Addiction, mindfulness, and acceptance. En Hayes, S. C., Jacobson, N. S., Follete, V. M y Dougher, M. J. (Eds) Acceptance and change: content and context in psychotherapy. Reno: Context Press.

Marlatt, G. A. (2011) Prefacio. En Bowen, S., Chawla, N. y Marlatt, G. A. (Eds) Mindfulness-Based Relapse Prevention for addictive behaviors. A clinician's guide. New York: The Guilford Press.

Miró, M. T., Perestelo-Pérez, L., Pérez, J., Rivero, A., González, M., De la Fuente, J. y Serrano, P. (2011) Eficacia de los tratamientos basados en mindfulness para los trastornos de ansiedad y depresión: una revisión sistemática. Revista de Psicopatología y Psicología Clónica, 6 (1), 1-14.

Morgan, W. D. y Morgan, S. T. (2005) Cultivating Attention and empathy. En Germer, C. K., Siegel, R. D. y Fulton, P. R. (Eds) Mindfulness and psychotherapy. New York: The Guilford Press.

Reiman, J. (1985) The impact of meditative attentional training on measures of select attentional parameters and on measures of client perceived counsellor empathy. Dissertation Abstracts International, 46, 15-69.

Roemer, L. y Orsillo, S. M. (2007) An open trial o fan acceptance-based behavior therapy for generalizad anxiety disorder. Behavior Therapy, 38, 72-85.

Segal, Z. V., Williams, J. M. G. y Teasdale, J. D. (2002) Mindfulness-Based Cognitive Therapy for depression. A new approach to preventing relapse. New York: The Guilford Press.

Sevillá, J. y Pastor, C. (1997) Toma de decisiones terapéuticas. Cómo decidir el plan de intervención. Información Psicológica, 65, 26-30.

Siegel, R. D., Germer, K. y Olendzki, A. (2011) Mindfulness: ¿Qué es? ¿Dónde surgió? En Didonna, F. (Ed), Manual clínico de mindfulness. Bilbao: Editorial Desclée de Brouwer.

Skinner, B. F. (1957) Verbal behavior. New York: AppletonCentury-Crofts.

Torneatto, T. y Nguyen, L. (2007).Does mindfulness medi- 
tation improve anxiety and mood syntoms? A review of the controlled research. La Revue canadienne de psychiatrie, 52, 4, 260-266.

Valentine, E. y Sweet, P. (1999) Meditation and attention: a comparison of the effects of concentrative and mindfulness meditation on sustained attention. Mental Health, Religion and Culture, 2, 1, 59-70.

Wells, A. (2009) Metacognitive Therapy for Anxiety and Depression. New York: The Guilford Press.

Wells, A. y Matthews, G. (1994) Attention and emotion: a clinical perspective. Hove: Erlbaum.

Wells, A. y Matthews, G. (1996) Modelling cognition in emotional disorder: the S-REF model. Behaviour Research and Therapy, 34, 881-888.

Wilson, K. G. y Luciano, M. C. (2002) Terapia de Aceptación y Compromiso (ACT). Un tratamiento orientado a los valores. Madrid: Pirámide.

Witkiewitz, K., Marlatt, G. A. y Walter, D. (2005) Mindfulness-based relapse prevention for alcohol and substance use disorders. Journal of Cognitive Psychotherapy, 19, 211-228.

Zgierska, A., Rabago, D., Chawla, N., Kushner, K., Kohler, R. y Marlatt, A. (2009) Mindfulness Meditation for Substance Use Disorders: A Systematic Review. Journal of Substance Abuse, 30, 266-294.

Fecha de recepción: 21/02/2013

Fecha de aceptación: 18/09/2013 